\title{
Twin pregnancy with a partial hydatidiform mole and a coexistent live fetus. Diagnostic and therapeutic dilemmas. A case report and the review of literature
}

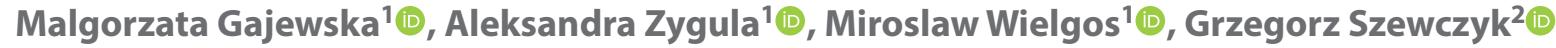 \\ ${ }^{1} 1^{\text {st }}$ Department of Obstetrics and Gynecology, Medical University of Warsaw, Poland \\ ${ }^{2}$ Center for Gestational Trophoblastic Disease, Institute of Mother and Child, Warsaw, Poland
}

\begin{abstract}
Objectives: We report the case of a twin pregnancy with a partial hydatidiform mole and a coexistent live fetus diagnosed in a 28-year-old primipara at 15 weeks of gestation and discuss the problems associated with the ultrasound diagnosis, histopathological examination of molar tissue samples and treatment.

Material and methods: A systematic research of the literature was conducted in PubMed database and Cochrane Library, including case reports and case series. A new case was also discussed. We collected data regarding the patient's serum human chorionic gonadotropin (hCG) level, initial symptoms, diagnosis and treatment.

Results: Most of the cases reported in the literature are those of a multiple pregnancy with complete hydatidiform mole (CHM) and a coexistent live fetus. The coexistence of a twin pregnancy with partial hydatidiform mole (PHM) and a live fetus in two separate amniotic sacs is extremely rare as a partial mole usually causes miscarriage of early pregnancy. Ultrasound is an important diagnostic tool, but the correct diagnosis is made only in $68 \%$ of cases. With further histological assessment of molar specimens and biochemical assays, the rates of correct early diagnoses should increase contributing to early therapeutic decisions and fewer adverse events.

Conclusions: The diagnosis, management, and monitoring of this condition will remain challenging because of its rarity. Because of that, all cases of a suspected multiple pregnancy with a hydatidiform mole and a coexistent live fetus should be referred to and managed at a tertiary center which specializes in the diagnosis and treatment of gestational trophoblastic disease.
\end{abstract}

Key words: hydatidiform mole; twin pregnancy; ultrasound; histopathological examination

Ginekologia Polska 2020; 91, 10: 589-594

\section{INTRODUCTION}

The coexistence of a hydatidiform mole with a normal fetus is extremely rare with an incidence of $1 / 20000$ to $1 / 100,000$ pregnancies [1-4]. In most cases, this is a complete hydatidiform mole (CHM). Partial hydatidiform mole (PHM) coexistent with a live fetus, in two separate amniotic sacs, is even less frequent since a partial molar pregnancy usually ends in early intrauterine death and miscarriage [5]. Diagnosis is by ultrasound, mostly in the second trimester, usually between 12 and 15 weeks [1,3]. Even with advanced ultrasound technologies, correct diagnosis is made in approximately $68 \%$ of cases and first-trimester diagnoses are rare. Assessment of human chorionic gonadotropin (hCG) levels are less reliable in a multiple pregnancy. Magnetic resonance imaging (MRI) is useful in the diagnosis of a molar pregnancy with a coexisting live fetus as it visualizes two amniotic sacs and a separate normal placenta and additionally allows assessment for myometrial infiltration and parametrial involvement [6].

There are three possible variations of a twin molar pregnancy with a coexistent live fetus: 1 . A dizygotic twin pregnancy with a complete mole and a live fetus of diploid karyotype; 2. A dizygotic twin pregnancy with a partial mole and a live diploid fetus in two separate amniotic sacs; 3. A monozygotic twin pregnancy with a partial mole and a live triploid fetus in a second amniotic sac [5].

This paper presents a case of a twin pregnancy with a partial hydatidiform mole and a coexistent live fetus and discusses problems associated with the ultrasound diagnosis, histopathological examination of molar tissue samples, and treatment. 


\section{Case report}

A 28-year-old primipara at 15 weeks of twin pregnancy was referred to the $1^{\text {st }}$ Department of Obstetrics and Gynecology, Medical University of Warsaw from an antenatal clinic at her local hospital with a suspected hydatidiform mole and a coexistent live fetus in a second amniotic sac.

\section{Pre-referral medical and obstetric history}

The age at menarche 13 years. Before pregnancy, the patient received thyroid hormone replacement therapy for hypothyroidism. She had been trying to conceive for two years and ovulation induction was used in the last four cycles before conceiving. At 12 weeks of gestation, she was admitted to hospital with ovarian hyperstimulation syndrome. An ultrasound examination showed in the uterine cavity one live fetus whose measurements were consistent with gestational age of 11 weeks and five days. Seen behind the uterus, the ovaries were enlarged (the right measured $94 \times 57 \times 40 \mathrm{~mm}$ and the left $94 \times 54 \times 41 \mathrm{~mm}$ ) with numerous follicles. There was some free fluid behind the uterus and between the coils of the intestine. There were no abnormalities detected on an abdominal ultrasound. The patient was prescribed anticoagulants and on day five was discharged home in good general condition. A first-trimester ultrasound performed one day later showed a normal development of a singleton pregnancy. The ovaries were enlarged as previously. After one week, the patient was readmitted to hospital for vaginal spotting. An ultrasound examination confirmed fetal cardiac activity and detected over the internal os a haematoma measuring $26 \times 6 \mathrm{~mm}$. A threatened miscarriage was diagnosed. The patient was prescribed progestins and antihaemorrhagic medication with a follow-up visit in 10 days when she was seen by her obstetrician. An ultrasound examination ten days later detected in the posterior uterine wall a $75 \times 148 \mathrm{~mm}$ lesion with numerous fluid-filled spaces. The serum hCG level was over $1000,000 \mathrm{mIU} / \mathrm{mL}$. The patient was referred to our Department.

\section{On admission}

Good general condition. On speculum examination a slight cervical bleeding. The size of the uterus corresponded with 25 weeks of gestation. Blood pressure $140 / 80$, pulse $112 / \mathrm{min}$, temperature $37.3^{\circ} \mathrm{C}$. An ultrasound examination detected in the uterine cavity a live fetus $131 \mathrm{~g}$ in weight, normal placenta and a cystic-solid lesion measuring $118 \times 77 \times 70 \mathrm{~mm}$. The right ovary measured $100 \times 60 \times 58 \mathrm{~mm}$ and the left ovary $101 \times 55 \times 53 \mathrm{~mm}$.

Blood tests: $\mathrm{RBC} 3.49$ million $/ \mathrm{mm}^{3}, \mathrm{Hb} 11 \mathrm{~g} / \mathrm{dL}, \mathrm{Ht} 30.2 \%$, WBC 8,600/mm $\mathrm{mm}^{3}$, PLT $289000 / \mathrm{mm}^{3}$, hCG $1601660 \mathrm{mIU} / \mathrm{mL}$, ALT $77 \mathrm{U} / \mathrm{L}$, LDH $327 \mathrm{U} / \mathrm{L}$, creatinine $0.62 \mathrm{mg} / \mathrm{dL}$, potassium $4.2 \mathrm{mmol} / \mathrm{L}$, sodium $136 \mathrm{mmol} / \mathrm{L}$, TSH $<0.005 \mu \mathrm{lU} / \mathrm{L}$, free
FT3 $7.61 \mathrm{pg} / \mathrm{mL}$, free FT4 $2.85 \mathrm{ng} / \mathrm{dL}$, anti-TSH receptor antibodies < $0.8 \mathrm{IU} / \mathrm{L}$, anti-TPO antibodies $1192.8 \mathrm{U} / \mathrm{mL}$, INR 1.12 , prothrombin time $12.4 \mathrm{~s}$, prothrombin ratio (PR) $90 \%$.

A multiple pregnancy with a $\mathrm{CHM}$ and a coexistent live fetus was diagnosed. In view of exceedingly high hCG levels, clinical hyperthyroidism confirmed by laboratory investigations and features of liver injury, the prognosis for pregnancy outcome was considered to be poor and the pregnancy threatened the mother's life and health. Termination of pregnancy was planned after prior institution of beta-blockers. The patient gave her informed written consent to surgical uterine evacuation, uterine curettage and hysterectomy, if necessary. The procedure was performed under general anaesthesia with antibiotic prophylaxis. The cervix of the uterus was dilated with Hegar dilators up to No 20. The molar tissue was aspirated under ultrasound guidance and next the fetus and placenta were removed. As the bleeding continued, curettage of the uterine cavity was performed, and oxytocin was given to induce haemostasis. The blood loss was estimated at ca. $2,000 \mathrm{~mL}$. On the first postoperative day, the patient was well with normal vital signs. The uterus was well contracted with a moderate vaginal bleeding. Blood tests: RBC 3.05 million $/ \mathrm{mm}^{3}$,

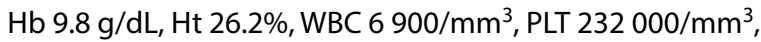
hCG 598350 mIU/mL, ALT 53 U/L, AST 34 U/L, LDH 318 U/L. Lactation was suppressed. On the third postoperative day a slight vaginal bleeding persisted, and the patient had no complaints. On pelvic examination the uterus remained well contracted and painless. An ultrasound examination showed an empty uterus and the same appearance of the ovaries as on previous scans. The patient was discharged home in good general condition and advised to have hCG levels monitored every seven days (on discharge the serum hCG was $160140 \mathrm{mlU} / \mathrm{mL}$ ) and to continue medication for lactation suppression and beta-blockers.

Three weeks later the patient collected the histopathology report of a complete hydatidiform mole. The hCG was $6,660 \mathrm{mlU} / \mathrm{mL}$ and close to the level measured seven days earlier $(6,672 \mathrm{mML})$. As the hCG levels failed to decrease, the patient was referred to her local oncology hospital where she was followed-up at one week intervals.

After a 3-month follow up, the patient was referred to the Center for Gestational Trophoblastic Disease at the Institute of Mother and Child. Based on the biochemical criteria (the plateau of hCG for the last four measurements) gestational trophoblastic neoplasia (GTN) was diagnosed. The molar specimens were re-examined at the Institute's Pathology Laboratory with the following findings: decidual and placental tissue fragments with features of oedematous degeneration of avascular villi, cistern-like villi present, mild focal atypia and partial degeneration of the trophoblast which corresponded with a partial hydatidiform mole. 
As there were two different histopathological diagnoses (complete vs partial hydatidiform mole), immunohistochemical staining for p57KIP2 was additionally performed which was positive in the trophoblastic cells and confirmed the diagnosis of a partial hydatidiform mole.

A transvaginal ultrasound examination visualized an anteverted uterus with the AP diameter of $54 \mathrm{~mm}$. The myometrial and endometrial echogenicity was homogenous, but in the posterior wall, adjacent to the internal os, a hyperechogenic lesion with visible vasculature, measuring $6 \times 5 \times 7 \mathrm{~mm}$ was detected. Both ovaries were normal. On Doppler ultrasonography the pulsatility index (PI) was 1.86 for the right uterine artery and 2.45 for the left uterine artery. There were no abnormalities detected on a chest $\mathrm{x}$-ray.

The patient was classified as low-risk according to the FIGO criteria (score 2; antecedent pregnancy - abortion, score 1 , pretreatment $\mathrm{hCG}$ between $10^{3}-10^{4} \mathrm{mIU} / \mathrm{L}$, score 1 ). MTX-FA (methotrexate/folinic acid) 8-day regimen, repeated every 2 weeks was prescribed (Tab. 1). The patient received chemotherapy in a total of 18 courses, including 3 courses of consolidation therapy. For the patient's convenience the treatment was administered in collaboration with her local hospital. The chemotherapy was well tolerated and occasional burning sensation in the oral cavity was relieved by symptomatic treatment, there were no cycle delays.

At present, the patient remains under close observation. The levels of hCG were monitored every 4 weeks for 6 months and then every 8 weeks for 12 months. The patient was advised to use effective contraception during that time.

\section{DISCUSSION}

The article presents difficulties in the diagnosis and treatment of a twin molar pregnancy with a coexistent normal live fetus. In the reported case, a molar pregnancy was first suspected as late as 15 weeks of gestation. Although the overall clinical and morphology picture was characteristic of a CHM, a PHM was identified by immunohistochemistry.

Table 1. Recommended single-agent chemotherapy regimens for gestational trophoblastic disease

MTX $0.4 \mathrm{mg} / \mathrm{kg}$ IM. or IV, on days 1, 2, 3, 4 and 5, repeat every 14 days MTX $50 \mathrm{mg} / \mathrm{m} 2 \mathrm{IM}$, repeat every 7 days

Dactinomycin $1.25 \mathrm{mg} / \mathrm{m} 2 \mathrm{IV}$, repeat every 14 days

Dactinomycin $12 \mu \mathrm{g} / \mathrm{kg}$ IV on days $1,2,3,4$ and 5 , repeat every 14 days

MTX $1 \mathrm{mg} / \mathrm{kg}$ (up to $70 \mathrm{mg}$ ) IM or IV, on days 1, 3,5 and 7, repeat every 14 days + FA $0.1 \mathrm{mg} / \mathrm{kg} \mathrm{IM}$ or IV, on days 2, 4, 6 and 8 , repeat every 14 days

MTX $50 \mathrm{mg} \mathrm{IM}$, on days 1, 3, 5 and 7, repeat every 14 days + FA $15 \mathrm{mg}$ IM or IV, 30h after the start of MTX administration

FA - folinic acid; IM - intramuscular; IV - intravenous; MTX - methotrexate
At present, an ultrasound examination is the main imaging tool used to establish the diagnosis. The 'Swiss-cheese pattern' placenta separate from a normal-appearing placenta is pathognomonic [6]. When the normal appearance of the placenta is not visualized, a complete hydatidiform mole with a coexistent live fetus (CHMCF) may be misdiagnosed as a singleton pregnancy with a partial mole and a live fetus in one amniotic sac. Since the prognosis for pregnancy outcome is different in each case, visualization of a normal placenta often adjacent to a large molar placenta is of key importance for the correct diagnosis [7, 8]. PHM is more difficult to diagnose than $\mathrm{CHM}$ even in a singleton pregnancy due to the absence of the characteristic ultrasound features. The differential diagnosis should include a subchorionic haematoma, with a similar cystic-solid appearance and a mild trophoblast pathology of placental mesenchymal dysplasia coexistent with Beckwith-Wiedemann syndrome in the fetus $[1,2,5]$.

Standard pathomorphologic assessment includes haematoxylin and eosin (H\&E) staining and distinguishing the type of hydatidiform mole by morphology. In each case of a molar pregnancy a second opinion should be sought from another expert of pathomorphology laboratory, as we did in the reported case. Immunohistochemical staining for the protein p57KIP2, which is the product of the CDKNIC gene, is a reliable diagnostic tool. As CDKNIC is paternally imprinted and maternally expressed, the p57 staining is absent in CHM which lacks a maternal genome [9]. In the reported case, CHM was initially diagnosed by morphology alone and after reassessment of the molar tissue specimens at a tertiary medical center, the final diagnosis of PHM was established, confirmed by positive staining for p57KIP2.

The coexistence of a molar pregnancy and a live normal fetus carries a risk of severe fetal and maternal complications. The most common maternal complications include severe haemorrhage leading to anaemia, severe preeclampsia, hyperthyroidism and thromboembolic disorders [1, 4-6, 8]. Also common are such events as fetal growth retardation, intrauterine death, miscarriage or premature birth $[1,4,7,10]$. Most of the cases reported in the literature are those of a multiple pregnancy with $\mathrm{CHM}$ and a coexistent live fetus. The coexistence of a multiple pregnancy with PHM and a live fetus in two separate amniotic sacs is extremely rare as a partial mole usually causes miscarriage of early pregnancy (10-20\% of early spontaneous abortions) $[3,11]$.

The reported patient had severe hyperthyroidism and features of liver injury which were indications for pregnancy termination. In the past, the diagnosis of a molar pregnancy with a coexistent live fetus was an indication for an immediate therapeutic termination $[4,8]$. Now, when a normal fetal development is confirmed by ultrasound and there are no maternal complications, analysis of the fetal karyotype is 
recommended. Women who decide to proceed with their pregnancy should be aware that the chances of a successful outcome are approximately $40 \%[1,2,6]$. A conservative approach is possible under close monitoring and when there are no maternal complications [3, 4, 6]. Decreasing hCG levels and absence of maternal complications are good predictors of a successful pregnancy outcome [12]. Abnormal fetal anatomy visualized by ultrasound or abnormal fetal karyotype are indications for termination. The risk for GTN in cases of a molar pregnancy with a coexistent live fetus ranges from 16 to $50 \%$ [1]. The development of invasive moles, choriocarcinomas or placental-site trophoblastic tumors has been reported [6]. The patient here reported developed GTN although she was finally diagnosed with PHM. The risk of GTN after surgical evacuation of CHM with a live fetus is significantly higher than the risk associated with evacuation of PHM in a multiple pregnancy (10-28\% vs 3-5\%) [5]. It is still unclear whether the continuation of pregnancy increases the risk for GTN [8]. According to many authors the duration of pregnancy has no effect on the development of GTN $[1,2,4,6,13]$. Also, there is no agreement concerning a more frequent occurrence of GTN after a twin pregnancy with CHM compared to a singleton pregnancy. Some authors found no differences in the incidence of GTN after a singleton complete molar pregnancy vs multiple molar pregnancy $[2,14]$. Steller et al., however, observed the development of GTN in as many as $55 \%$ of women with a molar pregnancy with a coexistent fetus [15]. A national collaborative study in Japan found a considerably higher rate of subsequent GTN development in patients with CHMCF. Heavy vaginal bleeding and severe preeclampsia are substantial risk factors for subsequent GTN [13].

The patient we report had ovulation induction for the last four cycles before conceiving. Some authors find a higher risk for twin molar pregnancies subsequent to ovulation induction [5]. In a case series reported by Giorgione et al. [1] one third of the patients had had ovulation induction. It is suspected that ovulation induction may be associated with the appearance of ova without nuclei and thus increase the risk of molar pregnancy [16].

The method of termination if the woman does not wish to proceed with her pregnancy or maternal medical conditions exclude the use of a conservative approach is another dilemma. Most controversial is the management of a molar pregnancy in the second trimester. Since the pregnancy is advanced, termination by vaginal evacuation may be associated with massive bleeding. When choosing hysterotomy abortion, extreme fetal immaturity must be taken into consideration. In the case here reported, the cervix was mechanically dilated, and the uterus evacuated, with an estimated blood loss of $2000 \mathrm{~mL}$. Vaisbuch et al. used a similar procedure at 16 weeks gestation in a patient with early onset severe preeclampsia and thyreotoxicosis [8]. Braga et al. reported termination by hysterotomy of a pregnancy with CHMCF at 15 weeks gestation, due to the worsening of the mother's clinical condition with hCG levels of $1881508 \mathrm{mIU} / \mathrm{mL}$ [7]. Braga commented that uterine evacuation by aspiration is not possible after the $12^{\text {th }}$ week of gestation due to the presence of a fetal skeleton. Also, the use of misoprostol is contraindicated as it may increase the likelihood of massive pulmonary thromboplastic embolization. After the first trimester, laparotomy and uterine evacuation by hysterotomy may be considered, especially when there are risk factors for adverse outcomes [7]. Close monitoring of hCG levels is mandatory after uterine evacuation of a molar pregnancy with a coexistent live fetus as it is after termination of a singleton pregnancy.

Differentiating of a complete mole from a partial mole is particularly important for choosing sufficient length of follow-up. With partial moles the risk of subsequent GTN is several-fold lower [17]. When a partial mole in a singleton pregnancy is evacuated, the patient needs to remain under observation for 4 weeks after the normalization of hCG. With a complete mole, hCG levels are monitored for up to 6 to 12 months, depending on the time of hCG normalization, within 8 weeks following evacuation or later than 8 weeks after evacuation.

Before starting the treatment of GTN, imaging studies should be performed to assess how advanced GTN is and to estimate the probability of single-agent chemotherapy failure. The patient here reported was classified as low-risk according to the FIGO criteria (score 2). MTX/FA 8-day regimen at a stable dose $50 \mathrm{mg} / 15 \mathrm{mg}$ was prescribed in view of its effectiveness and a manageable toxicity profile. FA given $30 \mathrm{~h}$ after MTX administration reduces MTX-associated haematological toxicity, but the time schedule of dosing must be strictly observed in order not to decrease the therapeutic effectiveness of MTX. Dactinomycin-based regimens have a less favourable adverse reaction profile with a higher incidence of nausea, vomiting and hair loss.

The hGG level was measured before each course of chemotherapy to assess response to treatment. During chemotherapy there is no need for imaging studies when the hCG levels gradually decrease. MTX/FA regimen was continued until hCG normalization and followed by three consolidation courses.

One important issue we encountered, was the correct assessment of hCG normalization which depends on the sensitivity of the laboratory test used. Several molecular variants of hCG present in serum include:

- intact hCG

- nicked intact hCG

- free $\beta$-subunit

- free $\alpha$-subunit 
- $\quad$ nicked free $\beta$-subunit

- $\beta$-subunit core fragment

In GTN, the percent ratios of free hCG $\beta$-subunit to total hCG vary depending on the degree of trophoblast differentiation from approximately $1 \%$ in PHM to $2.4 \%$ in CHM to $9 \%$ in choriocarcinoma. Carbohydrates account for approximately $30 \%$ of hCG mass and carbohydrate structure changes when they are synthesized by cancer cells. When the peptide chain is nicked, there is loss of biological activity and changes in the antigenicity of hCG. That is why a routine pregnancy test is less sensitive than the assays specifically designed to monitor hCG-secreting cancers. The use of hCG assays standardized to the World Health Organization (WHO) Fourth International Standard is recommended to minimize the risk of false negatives [18].

In the case here described, the hCG level before the $13^{\text {th }}$ course of chemotherapy, measured outside the reference center, was $4.3 \mathrm{mIU} / \mathrm{mL}$, which was considered as a negative result by the local center. The $\mathrm{hCG}$ level measured 14 days later at the reference center was $3.8 \mathrm{mIU} / \mathrm{mL}$ with the cut-off value of $1 \mathrm{mIU} / \mathrm{mL}$. A shared treatment decision made by the patient and her doctor was to continue regular hCG measurements but using a more sensitive assay and to proceed with chemotherapy until hCG levels returned to normal, which was observed before the $16^{\text {th }}$ course of chemotherapy. In line with the current recommendations for patients with low-risk GTN, three courses of consolidation chemotherapy were administered. A study from the UK and The Netherlands found two-fold lower relapse rates in low-risk GTN patients treated with three consolidation courses compared to patients treated with two consolidation courses [19]. After completing chemotherapy the patient must remain under observation and the hCG levels should be monitored with the measurements every month for six months after normalization, then every two months for up to 12 months post-normalization and finally every six months for up to 5 years. Patients are advised to use highly effective contraception in the first 12 months. In low-risk GTN the prognosis is good, there is a minor risk of recurrence and the complete remission rate is nearly $100 \%$.

To sum up, a multiple pregnancy complicated by a hydatidiform mole carries a risk of severe maternal and fetal adverse events. It should be emphasized that in the reported case the ultrasound scans and the clinical presentation strongly suggested the presence of a complete mole with a coexistent live fetus, but the diagnosis of a partial mole was ultimately made histologically. With further advances in ultrasound technologies, histological assessment of molar specimens and biochemical assays, the rates of correct early diagnoses should increase contributing to early therapeutic decisions and fewer adverse events. Considering their exceptionally low frequency, all cases of a suspected mul- tiple pregnancy with a hydatidiform mole and a coexistent live fetus should be referred to and managed at a tertiary center which specializes in the diagnosis and treatment of gestational trophoblastic disease.

\section{Conflict of interest}

All authors declare no conflict of interest.

\section{Funding}

There are no funding issues as this is a case study coupled with review.

\section{Ethical approval}

All the procedures performed in this study were in accordance with the ethical standards of Ethics Committee of Warsaw Medical University and with the 1964 Helsinki Declaration and its later amendments or comparable ethical standards. The publication of this case report got permission of the patient.

\section{Informed consent}

Informed consent was obtained from the patient for publication of this article.

\section{REFERENCES}

1. Giorgione V, Cavoretto P, Cormio G, et al. Prenatal Diagnosis of Twin Pregnancies with Complete Hydatidiform Mole and Coexistent Normal Fetus: A Series of 13 Cases. Gynecol Obstet Invest. 2017; 82(4): 404-409, doi: 10.1159/000448139, indexed in Pubmed: 27522447.

2. Freis A, Elsässer M, Sohn C, et al. Twin Pregnancy with One Fetus and One Complete Mole - A Case Report. Geburtshilfe Frauenheilkd. 2016; 76(7): 819-822, doi: 10.1055/s-0042-109398, indexed in Pubmed: 27453586.

3. Ingec $M$, Borekci $B$, Altas $S$, et al. Twin pregnancy with partial hydatidiform mole and coexistent normal fetus. J Obstet Gynaecol. 2006; 26(4): 379-380, doi: 10.1080/01443610600618747, indexed in Pubmed: 16753704.

4. Peng HH, Huang KG, Chueh HY, et al. Term delivery of a complete hydatidiform mole with a coexisting living fetus followed by successful treatment of maternal metastatic gestational trophoblastic disease. Taiwan J Obstet Gynecol. 2014; 53(3): 397-400, doi: 10.1016/j. tjog.2013.02.005, indexed in Pubmed: 25286799.

5. Gupta K, Venkatesan B, Kumaresan M, et al. Early Detection by Ultrasound of Partial Hydatidiform Mole With a Coexistent Live Fetus. WMJ. 2015; 114(5): 208-11; quiz 212, indexed in Pubmed: 26726342.

6. Imafuku H, Miyahara Y, Ebina $Y$, et al. Ultrasound and MRI Findings of Twin Pregnancies with Complete Hydatidiform Mole and Coexisting Normal Fetus: Two Case Reports. Kobe J Med Sci. 2018; 64(1): E1-E5, indexed in Pubmed: 30282891.

7. Braga $A$, Obeica $B$, Werner $H$, et al. A twin pregnancy with a hydatidiform mole and a coexisting live fetus: prenatal diagnosis, treatment, and follow-up. JUltrason. 2017; 17(71): 299-305, doi: 10.15557/JoU.2017.0044, indexed in Pubmed: 29375907.

8. Vaisbuch E, Ben-Arie A, Dgani R, et al. Twin pregnancy consisting of a complete hydatidiform mole and co-existent fetus: report of two cases and review of literature. Gynecol Oncol. 2005; 98(1): 19-23, doi: 10.1016/j. ygyno.2005.02.002, indexed in Pubmed: 15963812.

9. Gupta $M$, Vang R, Yemelyanova AV, et al. Diagnostic reproducibility of hydatidiform moles: ancillary techniques (p57 immunohistochemistry and molecular genotyping) improve morphologic diagnosis. Am J Surg Pathol. 2012; 36(3): 443-453, doi: 10.1097/PAS.0b013e31823b13fe, indexed in Pubmed: 22245958.

10. Sebire NJ, Foskett M, Paradinas FJ, et al. Outcome of twin pregnancies with complete hydatidiform mole and healthy co-twin. Lancet. 2002; 
359(9324): 2165-2166, doi: 10.1016/S0140-6736(02)09085-2, indexed in Pubmed: 12090984.

11. Sun $\mathrm{Cj}$, Zhao Yp, Yu S, et al. Twin pregnancy and partial hydatidiform mole following in vitro fertilization and embryos transfer: a novel case of placental mosaicism. Chin Med J (Engl). 2012; 125(24): 4517-4519, indexed in Pubmed: 23253730.

12. Bristow RE, Shumway JB, Khouzami AN, et al. Complete hydatidiform mole and surviving coexistent twin. Obstet Gynecol Surv. 1996; 51(12): 705-709, doi: 10.1097/00006254-199612000-00002, indexed in Pubmed: 8972493.

13. Matsui $H$, Sekiya $S$, Hando $T$, et al. Hydatidiform mole coexistent with a twin live fetus: a national collaborative study in Japan. Hum Reprod. 2000; 15(3): 608-611, doi: 10.1093/humrep/15.3.608, indexed in Pubmed: 10686205

14. Niemann I, Sunde L, Petersen LK. Evaluation of the risk of persistent trophoblastic disease after twin pregnancy with diploid hydatidiform mole and coexisting normal fetus. Am J Obstet Gynecol. 2007; 197(1): 45.e1-45.e5, doi: 10.1016/j.ajog.2007.02.038, indexed in Pubmed: 17618752.

15. Steller MA, Genest DR, Bernstein MR, et al. Clinical features of multiple conception with partial or complete molar pregnancy and coexisting fetuses. J Reprod Med. 1994; 39(3): 147-154, indexed in Pubmed: 8035369.

16. Bruchim I, Kidron D, Amiel A, et al. Complete hydatidiform mole and a coexistent viable fetus: report of two cases and review of the literature. Gynecol Oncol. 2000; 77(1): 197-202, doi: 10.1006/gyno.2000.5733, indexed in Pubmed: 10739712.

17. Coyle C, Short D, Jackson L, et al. What is the optimal duration of human chorionic gonadotrophin surveillance following evacuation of a molar pregnancy? A retrospective analysis on over 20,000 consecutive patients. Gynecol Oncol. 2018; 148(2): 254-257, doi: 10.1016/j. ygyno.2017.12.008, indexed in Pubmed: 29229282.

18. Whittington J, Fantz CR, Gronowski AM, et al. The analytical specificity of human chorionic gonadotropin assays determined using WHO International Reference Reagents. Clin Chim Acta. 2010; 411 (1-2): 81-85, doi: 10.1016/j.cca.2009.10.009, indexed in Pubmed: 19843470.

19. Lybol C, Sweep FC, Harvey R, et al. Relapse rates after two versus three consolidation courses of methotrexate in the treatment of low-risk gestational trophoblastic neoplasia. Gynecol Oncol. 2012; 125(3): 576-579, doi: 10.1016/j.ygyno.2012.03.003, indexed in Pubmed: 22410329. 